\title{
The Role of PET/CT in Evaluation of Recently Diagnosed Breast Cancer
}

EMAN G. AL-SAADAWY, M.D.; LOBNA K. SAKR, M.D. and REEM P. MOHAMED, M.Sc.

The Department of Radiodiagnosis, Faculty of Medicine for Girls, Al-Azhar University

\begin{abstract}
Background: Breast cancer is the most common non-skin cancer and the second leading cause of cancer related death in women. Breast cancer strikes women of all ages, races, ethnicities, socioeconomic strata, and geographic locales. Once breast cancer is diagnosed, the tumor stage has to be accurately determined before therapy chosen and the prognosis known.

Aim of Study: To investigate the ability of 18F-FDG $\mathrm{PET} / \mathrm{CT}$ to be used as a pre-operative assessment, staging and metastatic work up tool in patients with recently diagnosed breast cancer which is subsequently reflected in the management of breast cancer patients.
\end{abstract}

Patients and Methods: This study was carried out in Dokki Scan Radiology Center from April 2016 to October 2018 on a total number of 50 female patients with biopsy proved to have cancer breast. The age of the patients ranged from 27 to 78 years, and the average was 56.98. All exams were done on the PET/CT scanner Phillips Gemini \& Siemens time of flight $64 \& 20$ machines respectively.

Results: Whole-body 18F-FDG PET/CT plays an important role in the assessment of breast cancer patients particularly as it helps detection of the extent of the disease in the body, improves staging of breast cancer patients which significantly changes the therapeutic management of these patients.

Conclusion: PET/CT proved to be an efficient, accurate and non-invasive imaging technique in detecting metastatic disease with subsequent modification of the initial patient staging management planes.

Key Words: Cancer breast - CT imaging - PET/CT imaging - Staging.

\section{Introduction}

BREAST cancer is the most common type of skin cancer and the second leading cause of cancerrelated death in women. Breast cancer affects women of all ages, races, races, socioeconomic classes and geographical locations [1]

Correspondence to: Dr. Reem Prince Mohamed, E-Mail: reemprince@hotmail.com
Cancer regulation is necessary in determining the choice of treatment, as well as patient diagnosis and survival opportunities [2] .

Mammography has been shown to be accurate for breast cancer detection only in some specific cases, such as patients with severe breast cancer or large architectural deformities or extensive scars from previous biopsies [3]

Magnetic Resonance Imaging (MRI) can provide detailed information on the size and extent of breast cancer and has additional value for the evaluation of multi-focal and/or multicenter tumors [3].

However, conventional imaging cannot accurately detect the involvement of the axillary lymph node and/or the presence of distant metastases, which significantly changes the therapeutic management of these patients. The 18F-FDG PET/CT body has proven to be an effective means of imaging malignant tumors [3].

Moreover, the limited specificity of PET that's due to the increased glucose metabolic activities of benign tumors and inflammatory tissues (such as those of tuberculosis) can be partially overcome by PET/CT. Therefore, the $18 \mathrm{~F}-\mathrm{FDG} \mathrm{PET} / \mathrm{CT}$ would be more informative for pre-therapeutic staging of the entire body in a single examination [4].

Positron emission tomography (FDG) $18 \mathrm{~F}$ fluorose-2-Dioxide (PET) fused with CT provides the ability to combine functional and morphological information into a single study and thus becomes a powerful imaging tool for diagnosis, regulation of various malignant neoplasms, including breast cancer [5] 


\section{Aim of the work:}

The aim of the study is to investigate the ability of 18F-FDG PET/CT to be used as a pre-operative assessment, staging and metastatic work up tool in patients with recently diagnosed breast cancer which is subsequently reflected in the management of breast cancer patients.

\section{Patients and Methods}

This is study carried out in Dokki Scan Radiology Center from April 2016 to October 2018 for patients who have been recently diagnosed with cancer breast and were coming to perform PET/CT scans for staging, pre-operative or pre-therapeutic assessment.

A total number of 50 female patients were included in the study and they were biopsy proved to have cancer breast. The age of the patients ranged from 27 to 78 years, and the average was 56.98 .

All exams were done on the PET/CT scanner Phillips Gemini \& Siemens time of flight 64 \& 20 machines respectively.

Inclusion criteria: The study included patients with recently diagnosed breast cancers were included in this study.

\section{Exclusion criteria:}

The study excluded patients who received prior therapy or underwent previous operations for cancer breast or patients with blood glucose level $>300 \mathrm{mg} /$ $\mathrm{dl}$ at the time of the study.

\section{Imaging:}

All data were acquired with a combined PET/CT in-line system (Phillips Gemini 64 \& Siemens 20 time of flight machines). This dedicated system integrates a PET scanner with a multi-section helical CT scanner and permits the acquisition of co-registered CT and PET images in one session.

\section{Imaging protocol:}

\section{I- Patient preparation:}

All patients were asked to fast for six hours prior to scan. All metallic items were removed from the patient, including, pants with zipper, bra, belts, bracelets, etc. and the patient were given gown to wear. An intravenous (I.V) cannula was inserted in the patient's arm for administration of 18 F-FDG. The patients were instructed to avoid any kind of strenuous activity prior to the examination and following injection of the radioisotope to avoid physiologic muscle uptake of FDG and the patient was asked to void prior to scanning.

In case of diabetic patients; serum glucose was routinely measured prior to $18 \mathrm{~F}-\mathrm{FDG}$ injection, and fasting levels were 70-180ng/dl.

Diabetic patients should not have regular insulin administered subcutaneously within four hours of having FDG administered. We did not inject insulin usually to reduce blood glucose level.

Our strategies for decreasing brown fat were; providing a controlled-temperature (warm) environment for patients before $18 \mathrm{~F}-\mathrm{FDG}$ injection and high-fat, low-carbohydrate, protein-permitted diet before the examination.

\section{II- Dosage administration:}

10-20mCi (370MBq; approximate dose to patient, $3-5 \mathrm{MBq} / \mathrm{Kg}$ ) $18 \mathrm{~F}-\mathrm{FDG} 45-90$ minutes before examination. This period is referred to as the uptake phase and is the necessary amount of time for the FDG to be adequately bio-distributed and transported into the patient's cells. Patients were asked to rest in a quiet room, devoid of distractions, and they were also asked to keep their movements, including talking, at an absolute minimum. This minimizes physiologic uptake of FDG into skeletal muscle, which can confound interpretation of the scan. Patients should be comfortable and relaxed.

\section{III- Patient position:}

The patients were positioned in a comfortable head fixation in supine position with their arms raised above their heads.

\section{$I V$ - Examination time \& technique:}

We performed low dose non enhanced CT scan first, then a whole body PET study. Diagnostic enhanced whole body CT scan was then performed. The whole study took approximately 20-30 minutes.

For a typical whole body PET/CT study (brain, neck, chest, abdomen, and pelvis), scanning began from the skull base and extended caudally to the level of the upper thighs. The total length of CT coverage was an integral number of bed positions scanned during acquisition of PET data. The study was performed with the patient breathing quietly. Typical scanning parameters would be a col-limator width of $5.0 \mathrm{~mm}$, pitch of 1.5 , gantry rotation time of $0.8 \mathrm{~second}$, and field of view of $50 \mathrm{~cm}$. The helical data are retrospectively reconstructed at $1.0 \mathrm{~mm}$ intervals.

The contrast enhanced helical CT was performed following the PET study performance, 
using $125 \mathrm{~mL}$ of a low-osmolarity iodinated contrast medium using a power injector.

\section{VI- PET technique:}

Approximately six to seven bed positions are planned in the three-dimensional acquisition mode for scanning the entire patient with 3-5 minute acquisition at each bed position.

\section{VII- PET/CT fusion:}

Hundreds of transaxial PET and CT images were first reconstructed. These are then reformatted into coronal and sagittal images to facilitate image interpretation. For each of these sets of PET and CT images, corresponding "fusion" images, combining the two types of data, also were generated.

The whole acquisition time for an integrated PET/CT scan was approximately $25 \mathrm{~min}$. PET image data sets were reconstructed using CT data for attenuation correction and co-registered images were displayed using special software.

\section{Images interpretation:}

All CT examinations were analyzed by experienced radiologists and all PET/CT examinations were analyzed separately by a consensus of at least two experienced observers of nuclear medicine physicians and radiologists.

A Region of Interest (ROI) of 5.0 to $10.0 \mathrm{~mm}$ was placed manually over the area of maximal activity on slices with the clearest definition of the tumor mass and in the adjacent slices and the SUV was calculated.

The CT and fused PET/CT images were evaluated regarding the primary tumor and the presence of lymph nodes and distant metastases.

An increase in glucose uptake to a level greater than that in the surrounding tissue (more than the mediastinal blood pool in the chest and more than the background activity in the rest of the body) at qualitative analysis or a standard glucose uptake value of more than 2.5 were considered to characterize pathological process.

The possibility of nodal or distant metastatic disease spread was considered when lesions were clearly present on PET/CT images according to the combined morphologic CT and 18F-FDG uptake criteria. Abnormal 18F-FDG uptake was defined as radiotracer accumulation outside the normal anatomic structures and of greater intensity than background activity, excluding the known sites of physiologic FDG uptake.
For the analysis, the lymph node chains were grouped into axillary, contra-lateral axillary, internal mammary, hilar and mediastinal as well as abdominal and pelvic lymph nodes. The findings for each of these sites were graded as positive or negative for tumor infiltration.

In CT, lymph node assessment was based on size: Nodes with a short-axis diameter greater than $10.0 \mathrm{~mm}$ were defined as pathologic. Furthermore, the presence of necrosis within a lymph node was considered a sign of malignancy, regardless of node size. Also, the preserved fatty hilum of the lymph node was considered to be benign regardless of node size.

In PET/CT, lymph nodes with increased glucose uptake were considered positive for metastatic spread even when they were smaller than $1.0 \mathrm{~cm}$ in short-axis diameter. PET-negative lymph nodes were characterized as benign, even when they were larger than $1.0 \mathrm{~cm}$ in short-axis diameter.

Calcified lymph nodes were considered benign regarding the degree of FDG uptake.

The following sites were evaluated for distant metastases: Lung, visceral organs (liver, spleen \& adrenal glands), brain and bone. The findings for each of these sites were graded as positive or negative for tumor infiltration.

Lung nodules $5.0 \mathrm{~mm}$ in patients should be considered as positive if FDG uptake is greater than the mediastinal blood pool. Metastasis cannot be excluded in lung nodules $>0.5 \mathrm{~cm}$.

Hepatic or splenic lesions should be considered as positive if their uptake is higher than or equal to that of the liver or spleen, and negative if their uptake is lower than that of the liver and spleen.

The assessment of adrenal gland lesions was done according to a certain algorithm as follows: If the density of the lesion is less than $10.0 \mathrm{HU}$, it is considered as benign and if more than $10.0 \mathrm{HU}$, we assess SUV max of the lesion. If SUV max less than 3.1, it is considered benign and if more than 3.1 , it is considered malignant.

If there was a clearly multifocal increase in FDG uptake in the bone marrow, the patient was considered as PET positive.

\section{Statistical analysis:}

Data were statistically described in terms of range, mean \pm Standard Deviation $( \pm S D)$, median, frequencies (number of cases) and percentages when appropriate. Comparison of quantitative 
variables between the study groups was done using Kruskal Wallis Analysis of Variance (ANOVA) test. For comparing categorical data, Chi square $\left(\mathrm{X}^{2}\right)$ test was performed. Exact test was used instead when the expected frequency is less than 5 .

$p$-values less than 0.05 was considered statistically significant. All statistical calculations were done using computer programs Microsoft Excel 2010 (Microsoft Corporation, NY, USA) and SPSS (Statistical Package for the Social Science; SPSS Inc., Chicago, IL, USA) Version 15 for Microsoft Windows.

\section{Results}

Table (1): Patients' characteristics.

\begin{tabular}{lcccc}
\hline & Minimum Maximum & Mean & $\begin{array}{c}\text { Std. } \\
\text { Deviation }\end{array}$ \\
\hline - Age & 27 & 78 & 56.98 & 11.82 \\
- Weight & 49 & 130 & 78.05 & 14.53 \\
- Height & 130 & 178 & 161.05 & 8.254 \\
- Blood Glucose & 64 & 138 & 105.17 & 17.43 \\
- Dose of 18F- & 222 & 560 & 348.48 & 68.47 \\
$\quad$ FDG injected & & & & \\
\hline
\end{tabular}

Table (2): The patients with detected breast cancer by PET/CT versus $\mathrm{CT}$.

\begin{tabular}{lll}
\hline \multirow{2}{*}{ Modality } & \multicolumn{2}{c}{ Patients } \\
\cline { 2 - 3 } & \multicolumn{1}{c}{$+\mathrm{ve}$} & \multicolumn{1}{c}{$-\mathrm{ve}$} \\
\hline $\mathrm{PET} / \mathrm{CT}$ & $50(98 \%)$ & $1(2 \%)$ \\
$\mathrm{CT}$ & $42(82.4 \%)$ & $9(17.6 \%)$ \\
\hline
\end{tabular}

Table (3): The PET/CT characteristics of breast cancer lesions.

\begin{tabular}{lcccc}
\hline & Minimum & Maximum & Mean & $\begin{array}{c}\text { Standard } \\
\text { Deviation }\end{array}$ \\
\hline Size & 1.0 & 11.0 & 3.03 & 1.83 \\
SUV $_{\text {max }}$ & 2.1 & 36.3 & 8.01 & 6.79 \\
\hline
\end{tabular}

Table (4): Showing the percentage of the different size of breast cancer lesions.

\begin{tabular}{lcc}
\hline Size & No. of cases & Percentage \\
\hline$\leq 2 \mathrm{~cm}$ & 14 & 28 \\
$>2 \mathrm{~cm}$ but $<5 \mathrm{~cm}$ & 32 & 64 \\
$>5$ & 4 & 8 \\
\hline
\end{tabular}

Axillary lymph nodes:

Axillary lymph nodes involvement was detected by combined PET/CT in 33 patients, while CT alone was able to detect infiltrated axillary lymph nodes in 23 patients.

On a lymph node count, combined PET/CT detected 115 infiltrated lymph nodes, with 87 lymph nodes detected by the CT alone.
Comparing the results of combined PET/CT with CT alone in detection of $p$-value of PET/CT over CT alone 0.001 .

Table (5): Showing the axillary lymph nodes involvement by $\mathrm{PET} / \mathrm{CT}$ versus $\mathrm{CT}$.

\begin{tabular}{|c|c|c|c|}
\hline \multirow{2}{*}{ Modality } & \multicolumn{2}{|c|}{ Patients } & \multirow{2}{*}{ (n) Lymph nodes } \\
\hline & +ve & $-\mathrm{ve}$ & \\
\hline PET/CT & $33(64.7 \%)$ & $18(35.3 \%)$ & 115 \\
\hline $\mathrm{CT}$ & $23(45 \%)$ & $28(55 \%)$ & 87 \\
\hline
\end{tabular}

Contralateral axillary lymph nodes: Contralateral axillary lymph node metastases were detected by combined PET/CT in 8 patients ( 29 lymph nodes), while CT alone was able to detected in 6 patients (17 lymph nodes) with $p$-value of combined PET/CT over CT alone 0.066 .

Table (6): Showing the contralateral axillary lymph nodes involvement by $\mathrm{PET} / \mathrm{CT}$ versus $\mathrm{CT}$.

\begin{tabular}{lccc}
\hline \multirow{2}{*}{ Modality } & \multicolumn{2}{c}{ Patients } & (n) Lymph nodes \\
\cline { 2 - 3 } & +ve & $-v e$ & \\
\hline PET/CT & $8(16.32 \%)$ & $41(83.68 \%)$ & 29 \\
CT & $6(12.25 \%)$ & $43(87.75 \%)$ & 17 \\
\hline
\end{tabular}

Internal mammary lymph nodes: Internal mammary lymph node metastases were detected by combined PET/CT in 9 patients (9 lymph nodes), while $\mathrm{CT}$ alone was able to detected in 4 patients (4 lymph nodes), with $p$-value of combined PET/CT over CT alone 0.025 .

Table (7): The internal mammary lymph nodes involvement by $\mathrm{PET} / \mathrm{CT}$ versus $\mathrm{CT}$.

\begin{tabular}{llcc}
\hline \multirow{2}{*}{ Modality } & \multicolumn{2}{c}{ Patients } & \\
\cline { 2 - 3 } & \multicolumn{1}{c}{ +ve } & (n) Lymph nodes \\
\hline PET/CT & $9(17.65 \%)$ & $42(82.35 \%)$ & 9 \\
CT & $4(7.84 \%)$ & $47(92.16 \%)$ & 4 \\
\hline
\end{tabular}

Other lymph nodes: Extra-axillary lymph nodes metastasis include cervical, mediastinal, hilar abdominal and pelvic lymph nodes. Combined $\mathrm{PET} / \mathrm{CT}$ found distant lymph node metastasis in 18 patients (145 lymph nodes), with 15 patients (86 lymph nodes) found to have positive lymph node metastasis by $\mathrm{CT}$ alone, with $p$-value of combined PET/CT over CT alone 0.005 .

Table (8): The other extra-axillary lymph nodes involvement by PET/CT versus CT.

\begin{tabular}{lccc}
\hline \multirow{2}{*}{ Modality } & \multicolumn{2}{c}{ Patients } & \\
\cline { 2 - 3 } & +ve & (n) Lymph nodes \\
\hline PET/CT & $18(35.3 \%)$ & $33(64.7 \%)$ & 145 \\
CT & $15(29.4 \%)$ & $36(70.6 \%)$ & 86 \\
\hline
\end{tabular}


These extra-axillary lymph nodes were found in the cervical group (in 8 patients), mediastinal (in 13 patients), hilar (in 9 patients) and pelviabdominal lymph nodes (in 5 patients).

Table (9): The site and percentage of the patients with extraaxillary lymph nodes involvement.

\begin{tabular}{lcc}
\hline Site & No. of cases & Percentage \\
\hline Cervical & 8 & 15.7 \\
Mediastinal & 13 & 25.5 \\
Hilar & 9 & 17.6 \\
Pelvi-abdominal & 5 & 9.8 \\
\hline
\end{tabular}

Pulmonary metastasis:

Out of 51 patients, combined PET/CT detected pulmonary metastasis in 7 patients. On the other side, CT alone detected 6 patients with pulmonary metastasis ( $p$-value of combined PET/CT over CT alone was 0.317 ).

Table (10): The pulmonary metastasis detected by PET/CT versus $\mathrm{CT}$.

\begin{tabular}{|c|c|c|}
\hline \multirow{2}{*}{ Modality } & \multicolumn{2}{|c|}{ Patients } \\
\hline & $+\mathrm{ve}$ & $-\mathrm{ve}$ \\
\hline PET/CT & $7(13.7 \%)$ & $44(86.3 \%)$ \\
\hline $\mathrm{CT}$ & $6(11.8 \%)$ & $45(88.2 \%)$ \\
\hline
\end{tabular}

\section{Visceral metastasis:}

Combined PET/CT has shown metastatic visceral disease in 9 patients (including the liver, spleen, omentum and suprarenal gland), while CT alone found the disease in 8 patients with $p$-value of combined PET/CT over CT alone was 0.18 .

Table (11): The visceral metastasis detected by PET/CT versus CT.

\begin{tabular}{|c|c|c|}
\hline \multirow{2}{*}{ Modality } & \multicolumn{2}{|c|}{ Patients } \\
\hline & $+\mathrm{ve}$ & $-\mathrm{ve}$ \\
\hline $\mathrm{PET} / \mathrm{CT}$ & $9(17.65 \%)$ & $42(82.35 \%)$ \\
\hline CT & $8(7.84 \%)$ & $43(84.31 \%)$ \\
\hline
\end{tabular}

Table (12): The sites of the visceral metastasis detected by combined PET/CT.

\begin{tabular}{lc}
\hline Site & No. of cases \\
\hline Liver & 8 \\
Spleen & 1 \\
Suprarenal gland & 1 \\
Omentum & 1 \\
\hline
\end{tabular}

Osseous deposits:

Out of 51 patients, combined PET/CT detected osseous deposits in 15 patients, with 13 patients were found to have osseous deposits by $\mathrm{CT}$ alone ( $p$-value of combined PET/CT over CT alone was $0.2)$.
Bone scan results were available for 8 patients of the positive patients detected by combined $\mathrm{PET} / \mathrm{CT}$, and they reveal 7 patients with osseous deposits, with one patient showing negative results for deposits (however further MRI study revealed metastatic bone disease).

Table (13): The osseous deposits detected by PET/CT versus CT.

\begin{tabular}{|c|c|c|}
\hline \multirow{2}{*}{ Modality } & \multicolumn{2}{|c|}{ Patients } \\
\hline & $+\mathrm{ve}$ & $-\mathrm{ve}$ \\
\hline PET/CT & $15(29.4 \%)$ & $36(70.6 \%)$ \\
\hline CT & $13(25.5 \%)$ & $38(74.5 \%)$ \\
\hline
\end{tabular}

TNM staging:

A- Local tumor staging (T):

Among 51 patients, combined PET/CT staged 17 patients as T1, 18 patients as T2, 15 patients as $\mathrm{T} 4$ with one patient showing no evidence of primary tumor.

Table (14): The T1 staging by PET/CT versus CT.

\begin{tabular}{lcccccc}
\hline & T0 & T1 & T2 & T3 & T4 & Total \\
\hline CT & 9 & 13 & 17 & 0 & 12 & 51 \\
PET/CT & 1 & 17 & 18 & 0 & 15 & 51 \\
\hline
\end{tabular}

Of the 14 patients who had T4 by combined $\mathrm{PET} / \mathrm{CT}, 10$ patients had ipsilateral satellite nodules, 2 patients with ipsilateral satellite nodules and skin edema/thickening, 1 patient with skin ulceration and 1 patient showing breast skin edema/thickening.

\section{B- Regional lymph nodes ( $N)$ :}

Regarding the combined PET/CT (N) staging, The $\mathrm{N}$ stage in 51 patients was as follows: N0 in 16 patients, $\mathrm{N} 1$ in 23 patients, $\mathrm{N} 2$ in 3 patients and N3 in 9 patients.

While CT alone showed N0 in 28 patients, N1 in 14 patients, $\mathrm{N} 2$ in 1 patient and finally N3 in 8 patients.

Table (15): $\mathrm{N}$ staging by PET/CT versus $\mathrm{CT}$.

\begin{tabular}{lccccc}
\hline & N0 & N1 & N2 & N3 & Total \\
\hline CT & 28 & 14 & 1 & 8 & 51 \\
PET/CT & 16 & 23 & 3 & 9 & 51 \\
\hline
\end{tabular}

Of the 9 patients with N3 stage, 4 patients had ipsilateral internal mammary and axillary lymph nodes involvement, 3 patients with involved ipsilateral supraclavicular lymph nodes, 1 patient with ipsilateral infra-clavicular lymph node and finally 1 patient with ipsilateral axillary and supraclavicular lymph nodes involvement. 


\section{$C$ - Distant metastasis (M):}

Of the 51 patients, combined PET/CT detected distant metastasis in 25 patients, while $\mathrm{CT}$ alone detected distant metastasis in 23 patients.

Table (16): M staging by PET/CT versus CT.

\begin{tabular}{lccc}
\hline & M0 & M1 & Total \\
\hline CT & 28 & 23 & 51 \\
PET/CT & 26 & 25 & 51 \\
\hline
\end{tabular}

\section{$D$ - Overall staging:}

Combined PET/CT staged 3 patients as stage IA, 10 patients as stage IIA, 7 patients as stage IIB, 2 patients as stage IIIA, 2 patients as stage IIIB, 2 patients as stage IIIC and 25 patients as stage IV.
Out of 51 patients, combined PET/CT upgraded the staging of 13 patients compared to initial CT staging.

Overall comparison between CT versus combined PET/CT:

Table (17): Staging of the involved patients.

\begin{tabular}{lc}
\hline Stages & No. of patients \\
\hline 0 & 0 \\
IA & 3 \\
IB & 0 \\
IIA & 10 \\
IIB & 6 \\
IIIA & 2 \\
IIIB & 3 \\
IIIC & 2 \\
IV & 25 \\
\hline
\end{tabular}

Table (18): Comparison between PET/CT versus CT alone.

\begin{tabular}{|c|c|c|c|c|c|}
\hline & \multicolumn{2}{|c|}{ CT } & \multicolumn{2}{|c|}{ PET/CT } & \multirow{2}{*}{$\begin{array}{c}p- \\
\text { value }\end{array}$} \\
\hline & Positive & Negative & Positive & Negative & \\
\hline Primary breast cancer & $42(82.4 \%)$ & $9 \quad(17.6 \%)$ & $50(98 \%)$ & $1(2 \%)$ & 0.002 \\
\hline Axillary lymph nodes & $23(45 \%)$ & $28(55 \%)$ & $33(64.7 \%)$ & $18(35.3 \%)$ & 0.001 \\
\hline Contralateral axillary lymph nodes & $6 \quad(12.25 \%)$ & $43(87.75 \%)$ & $8 \quad(16.32 \%)$ & $41(83.68 \%)$ & 0.066 \\
\hline Internal mammary lymph nodes & $4 \quad(7.84 \%)$ & $47(92.16 \%)$ & $9 \quad(17.65 \%)$ & $42(82.35 \%)$ & 0.025 \\
\hline Other lymph nodes & $15(29.4 \%)$ & $36(70.6 \%)$ & $18(35.3 \%)$ & $33(64.7 \%)$ & 0.005 \\
\hline Pulmonary metastasis & $6 \quad(11.8 \%)$ & $45(88.2 \%)$ & $7 \quad(13.7 \%)$ & $44(86.3 \%)$ & 0.317 \\
\hline Visceral metastasis & $8 \quad(7.84 \%)$ & $43(92.16 \%)$ & $9 \quad(17.65 \%)$ & $42(82.35 \%)$ & 0.18 \\
\hline Osseous deposits & $13(25.5 \%)$ & $38(74.5 \%)$ & $15(29.4 \%)$ & $36(70.6 \%)$ & 0.2 \\
\hline
\end{tabular}
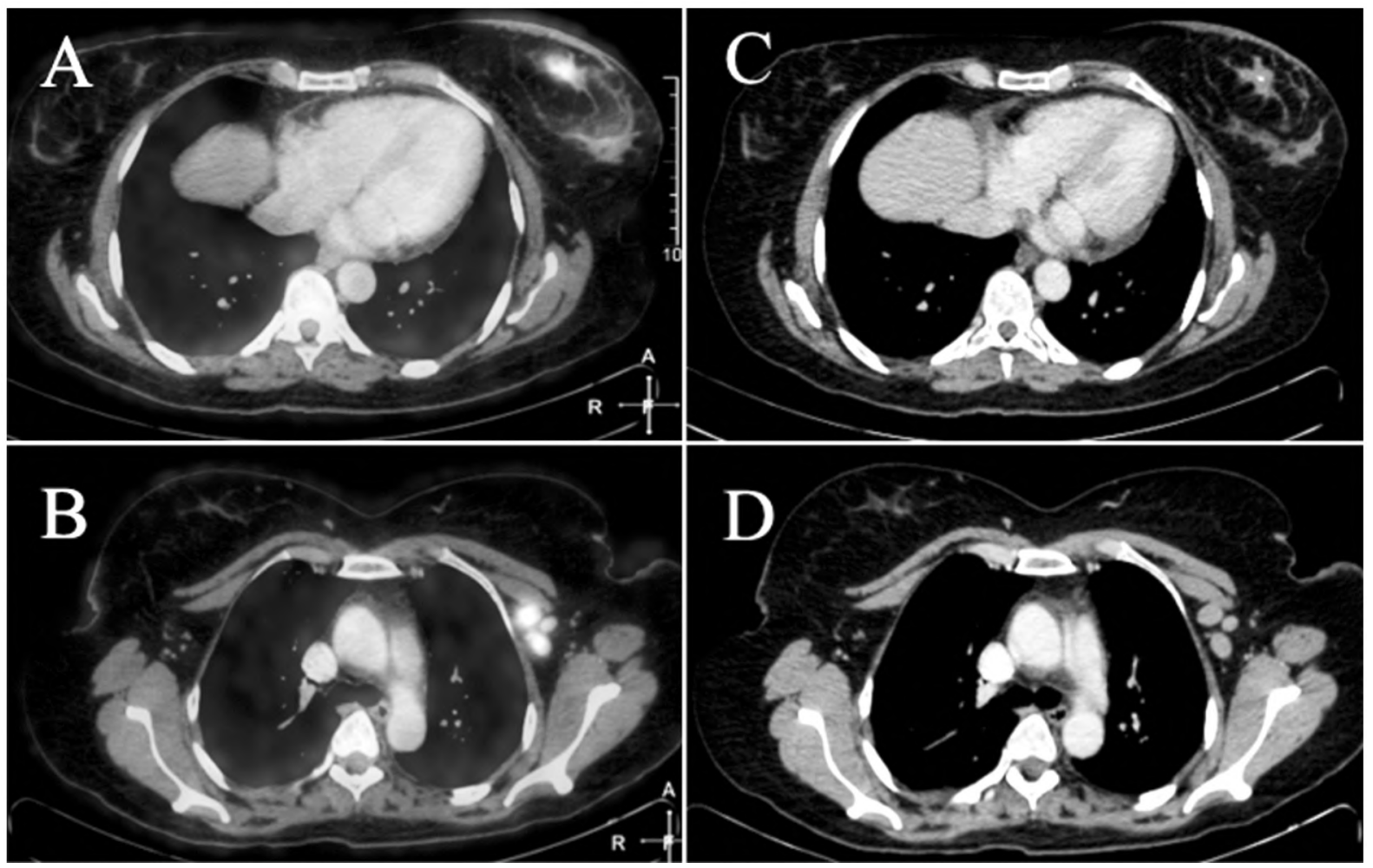

Fig. (1): 51 year old female patient with pathologically proved left breast cancer for pre-treatment assessment. (A \& C) Axial post contrast CT cuts, (B \& D) Axial combined PET/CT images. 


\section{Discussion}

Clinical evaluation of patients with cancer is critical to start and evaluate the effect of treatment. The results of CT can be better assessed with the help of additional functional information provided by FDG PET, particularly in the follow-up of cancer patients undergoing surgery, radiation therapy or chemotherapy [6]

FDG PET is superior to conventional CT in the aspects of initial cancer assessment and progression. Misleading results may result from fine metabolic results in FDG PET, which may be confused with natural physiological absorption. CT scans may allow detection of pathological sites for FDG accumulation [7].

The use of contrast enhanced CT in PET/CT helps in better anatomical localization and characterization of lesions but contrast enhanced pixels can potentially generate focal artifacts in the PET image with undesirable outcome particularly for tumor imaging [8].

We performed PET combined with low dose $\mathrm{CT}$ for attenuation correction and then performed contrast enhanced CT for image fusion. In accordance with Klausen et al., [9], we did not use the contrast enhanced CT for attenuation correction and thus we did not face the possible artifactual hot spots on the attenuation corrected image or quantitative over estimation of $18 \mathrm{~F}-\mathrm{FDG}$ activity as mentioned by Townsend et al., [10] .

In this study, contrast enhanced CT images were performed for the involved patients to ensure fully diagnostic CT data, and such strategy was in line with other studies as to Klausen et al., [9].

Performing diagnostic contrast enhanced CT provides a baseline exam in case that follow-up $\mathrm{CT}$ alone is to be used for monitoring the therapeutic response as the cost of the PET/CT exam may not be affordable for some patients [11].

There was only one patient in this study with elevated renal functions and thus the contrast enhanced diagnostic CT was not performed. There was no significant difficulty in detection of the involved mediastinal lymph nodes. However the main drawback was in the detection of the involved hilar lymph nodes as well as the hepatic focal lesions, which could be missed in the un-enhanced study and would be difficult to deicide precisely the affected segment.

The major aim of the study is to investigate the ability of 18F-FDG PET/CT to be used as a pre- operative and pre therapeutic assessment study. Several studies have assessed its role in modification of initial patients staging over the other conventional studies and it proved its superiority. Using PET/CT as metastatic work up tool is very crucial in accurate staging of the patients which is considered as the corner stone in the management plan of breast cancer patients.

In this study, regarding the assessment of the primary breast tumors in 50 patients, it has shown that combined PET/CT was superior to CT alone in detection of the primary breast cancer with sensitivity $98.11 \%$ compared to $81.7 \%$ for CT alone.

This was on agreement with previous studies by Klausen et al., [9] who have stated $97 \%$ sensitivity of PET/CT in identification of primary breast tumor. Moreover, Tatsumi et al., [4] have shown that PET/CT is preferable to PET or CT in the diagnosis of breast cancer. In a study performed on 60 patients by Fuster et al., [3], combined PET/CT was able to identify the primary tumor among the all patients.

Niikura et al., [12] have also reported that higher sensitivity of FDG-PET over CT alone with FDG PET sensitivity of $93 \%$ for newly diagnosed breast cancer, compared to $88 \%$ sensitivity of CT in patients with newly diagnosed disease.

Combined PET/CT missed only one patient with breast cancer, and that might be explained by the relatively dense patient breast parenchyma \& the small size of the lesion. This explanation was supported by study done by Vercher-Conejero et al., [13] who have shown high sensitivity (94\%) of combined PET/CT in breast tumors larger than $1 \mathrm{~cm}$, with reduction of sensitivity $(63 \%)$ among the breast cancer lesions smaller than $5 \mathrm{~mm}$.

In this study, detection of metastatic axillary lymph nodes was improved by combined PET/CT which detected metastatic axillary lymph nodes in 33 patients compared to 23 patients showing positive axillary involvement by CT alone (of PET/CT over CT alone 0.001).

In the current study, there was mismatch between CT \& combined PET/CT in 14 patients regarding the detection of metastatic axillary lymph nodes. In these patients combined PET/CT detected axillary lymph nodes involvement with negative results in CT alone. In 5 patients this mismatch causes no changes in the $\mathrm{N}$ staging, while in the other 9 patients this mismatch causes upstaging of 
$\mathrm{N}$ stage (8 patients from N0 to $\mathrm{N} 1$ and 1 patient from N0 to N3).

This mismatch can be explained by the small sub-centimetric lymph nodes, considered to be negative in $\mathrm{CT}$, and have shown high metabolic activity in combined PET/CT study.

In studies done by Vercher-Conejero et al., [13], and Fuster et al., [3], combined PET/CT have shown excellent specificity (90 100\%) and positive predictive value for detection of metastatic axillary lymph nodes with sensitivity (63 70\%).

We agreed with study done Tatsumi et al., [4], who have revealed superiority of combined PET/CT over CT alone in detection of metastatic axillary lymph nodes. Combined PET/CT detected positive axillary lymph nodes in 25 regions with 14 positive regions detected by CT alone.

In this study, detection of metastatic internal mammary lymph nodes was improved by combined PET/CT which detected metastatic internal mammary lymph nodes in 9 patients compared to 4 patients showing positive internal mammary chain involvement by CT alone (of PET/CT over CT alone 0.025).

In the current study, there was mismatch between CT \& combined PET/CT in 5 patients regarding the detection of metastatic internal mammary lymph nodes. In these patients combined PET/CT detected axillary lymph nodes involvement with negative results in CT alone. In 2 patients, this mismatch causes no changes in the $\mathrm{N}$ staging, while in the other 3 patients this mismatch causes upstaging of $\mathrm{N}$ stage (two patients from N0 to N2, and 1 patient from N0 to N3).

This result was in agreement with Tatsumi et al., [4], study which had proved FDG-PET/CT to be useful in detecting tumoral involvement of the internal mammary lymph nodes compared to the CT because the smaller lymph nodes sometimes produced equivocal or negative CT findings. Therefore, PET/CT may play an important role in predicting patient outcome, as it is known that patients with metastases to these lymph nodes have a significantly worse prognosis than those without tumoral involvement.

In our study, combined PET/CT appears superior over the CT alone in detection of the extraaxillary lymph nodes. Combined PET/CT found distant lymph node metastasis in 18 patients, with 15 patients found to have positive lymph node metastasis by $\mathrm{CT}$ alone, with of combined PET/CT over CT alone 0.005 . Contra-lateral axillary lymph node metastases were detected by combined $\mathrm{PET} / \mathrm{CT}$ in 8 patients, while $\mathrm{CT}$ alone was able to detected in 6 patients with of combined PET/CT over CT alone 0.066 we agree with Choi et al., [14] who have reported that combined PET/CT was able to localize extra-axillary lymph nodes involvement better than contrast enhanced CT.

Distant metastases from breast cancer are frequently found in the lungs, liver and bones. One advantage of whole-body PET/CT imaging over conventional imaging modalities is its ability to detect metastasis at different sites and organs during a single examination [15].

Out of 51 patients, combined PET/CT detected pulmonary metastasis in 7 patients, with 6 patients show pulmonary metastatic disease by $\mathrm{CT}$ alone. The one patient with mismatch was showing pulmonary nodule measuring about $1 \mathrm{~cm}$ in diameter with no other nodules, and it shows high metabolic activity in combined PET/CT study.

Combined PET/CT has shown metastatic visceral disease in 9 patients (11 sites including the liver, omentum, spleen and suprarenal gland), while $\mathrm{CT}$ alone found the disease in 8 patients with of combined PET/CT over CT alone was 0.18.

One patient with mismatch between combined PET/CT \& CT alone findings. In this patient combined PET/CT detected FDG-avid focal lesions within the right and left lobes with no definite CT correlate could be seen. That may be explained by the lack of contrast enhanced cuts in such patient, attributed to her elevated renal functions.

Out of 51 patients, combined PET/CT detected osseous deposits in 15 patients, with 13 patients were found to have osseous deposits by $\mathrm{CT}$ alone (of combined PET/CT over CT alone was 0.2).

Bone scan results were available for 8 patients of the positive patients detected by combined PET/CT, and they reveal 7 patients with osseous deposits, with one patient showing negative results for bony metastasis, however further MRI study revealed metastatic bone disease.

Overall, this study have demonstrated superiority of combined PET/CT over CT alone in detection of distant metastasis.

Study done by Groheux et al., [16] have also revealed that all patients with metastatic lung disease were detected by the means of combined PET/CT and chest imaging modalities, except for a patient with pleural effusion where PET/CT 
corrected the diagnosis that the patient had isolated pleural effusion, which was considered benign on CE-CT.

However, the PET/CT scan showed high nodular uptake in the effusion, which was interpreted as metastasis and later confirmed by pleural aspiration as malignant.

We agree with Bernsdorf et al., [17] who have found that PET/CT is a valuable tool to provide information on extra-axillary lymph node involvement, distant metastases and other occult primary cancers. In the initial stage assessment, pre-operative FDG-PET/CT has a substantial impact on staging and on clinical management and they suggest that PET/CT could be considered in the initial assessment of patients with newly diagnosed nodepositive early-stage breast cancer.

This was in line with study done by Choi et al., [14] stated that the overall sensitivity and specificity in detecting distant metastases of the 18F-FDG PET/CT were $100 \%$ and $96.4 \%$, respectively; whereas the sensitivity and specificity of the conventional imaging were $61.5 \%$ and $99.2 \%$, respectively.

They have also reported that combined PET/CT has higher sensitivity, specificity and accuracy than CT in detection of the bone metastasis. They have demonstrated that PET/CT is preferable in evaluating breast cancer lesions.

Moreover Groheux et al., [16] and Morris et al., [18] have shown that PET/CT is more effective in detection of metastatic bone disease than conventional imaging (including bone scan).

\section{Conclusion:}

Our study results show that combined PET/CT is a single valuable tool provides whole-body overview evaluation of recently diagnosed breast cancer patients, providing valuable information on axillary and extra-axillary lymph node involvement as well as distant metastases. PET/CT proved to be an efficient, accurate and non-invasive imaging technique in detecting metastatic disease with subsequent modification of the initial patient staging management planes.

\section{References}

1- POWER E.J., CHIN M.L. and HAQ M.M.: Breast cancer incidence and risk reduction in the hispanic population. Cureus., 10 (2), 2018.
2- BRIERLEY J., GOSPODAROWICZ M. and O'SULLIVAN B.: The principles of cancer staging. Ecancermedicalscience, 10, 2016.

3- FUSTER D., DUCH J., PAREDES P., et al.: Pre-operative staging of large primary breast cancer with $[18 \mathrm{~F}]$ Fluorodeoxyglucose positron emission tomography/computed tomography compared with conventional imaging procedures. J. Clin. Oncol., 26 (29): 4746-51, 2008.

4- TATSUMI M., COHADE C., MOURTZIKOS K.A., et al.: Initial experience with FDG-PET/CT in the evaluation of breast cancer. Eur. J. Nucl. Med. Mol. Imaging, 33: 254-62, 2006.

5- BORGATTI A., WINTER A.L., STUEBNER K., et al.: Evaluation of 18-F-fluoro-2-deoxyglucose (FDG) positron emission tomography/ computed tomography (PET/CT) as a staging and monitoring tool for dogs with stage-2 splenic hemangiosarcoma-A pilot study. PloS One, 12 (2): $\mathrm{e} 0172651,2017$

6- LIM HS Y., CHUNG T.W., KIM JK J.G., et al.: FDG PET/CT for the detection and evaluation of breast diseases: Usefulness and limitations. Radiographics, 27 (1): 197 213, 2007.

7- COCHET A., DYGAI-COCHET I., RIEDINGER J.M., et al.: 18F-FDG PET/CT provides powerful prognostic stratification in the primary staging of large breast cancer when compared with conventional explorations. Eur. J. Nucl. Med. Mol. Imaging, 41: 428-37, 2014.

8- PFANNENBERG C., ASCHOFF P., SCHANZ S., et al.: Prospective comparison of $18 \mathrm{~F}$-fluorodeoxyglucose positron emission tomography/computed tomography and whole-body magnetic resonance imaging in staging of advanced malignant melanoma. European Journal of Cancer, 43 (3): 557-64, 2007.

9- KLAUSEN T.L., MORTENSEN J., DE NIJS R., et al. Intravenous contrast-enhanced CT can be used for CTbased attenuation correction in clinical 111 In-octreotide SPECT/CT. EJNMMI Physics, 2 (1): 3, 2015.

10- TOWNSEND D.W., CARNEY J.P., YAP J.T., et al.: PET/CT today and tomorrow. The Journal of Nuclear Medicine, 45: 4, 2004.

11- RODRÍGUEZ-VIGIL B., GÓMEZ-LEÓN N., PINILLA I., et al.: PET/CT in lymphoma: Prospective study of enhanced full-dose PET/CT versus unenhanced low-dose PET/CT. Journal of Nuclear Medicine, 47 (10): 1643-8, 2006.

12- NIIKURA N., COSTELLOE C.M., MADEWELL J.E., et al.: FDG-PET/CT compared with conventional imaging in the detection of distant metastases of primary breast cancer. The Oncologist, 16 (8): 1111-9, 2001.

13- VERCHER-CONEJERO J., PELEGRÍ-MARTINEZ L., LOPEZ-AZNAR D., et al.: Positron emission tomography in breast cancer. Diagnostics, 5 (1): 61-83, 2015.

14- CHOI Y.J., SHIN Y.D., KANG Y.H., et al.: The effects of pre-operative 18F-FDG PET/CT in breast cancer patients in comparison to the conventional imaging study. Journal of Breast Cancer, 15 (4): 441-8, 2012.

15- LEE J.W., LEE S.M. and CHOI J.H.: 18 F-FDG PET/CT Findings in a Breast Cancer Patient with Concomitant 
Tuberculous Axillary Lymphadenitis. Nuclear Medicine and Molecular Imaging, 45 (2): 152-5, 2011.

16- GROHEUX D., GIACCHETTI S., ESPIÉ M., et al.: The yield of 18F-FDG PET/CT in patients with clinical stage IIA, IIB, or IIIA breast cancer: A prospective study. Journal of Nuclear Medicine, 52 (10): 1526-34, 2011.

17- BERNSDORF M., BERTHELSEN A.K., WIELENGA
V.T., et al.: Pre-operative PET/CT in early-stage breast cancer. Ann. Oncol., 23 (9): 2277-82, 2012.

18-MORRIS P.G., LYNCH C., FEENEY J.N., et al.: Integrated positron emission tomography/computed tomography may render bone scintigraphy unnecessary to investigate suspected metastatic breast cancer. Journal of Clinical Oncology, 28 (19): 3154, 2010.

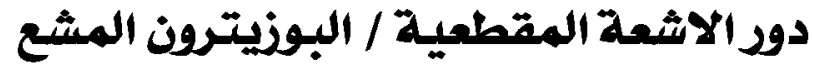

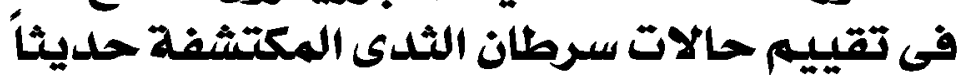

يعتبر سرطان الثىى أحد أنواع السرطانات غير الجلدية الاكتر شيوعاً والسبب الرئيسى الثانى اللوفاة المتعلقة بالسرطان لدى الأى النساء. يصيب

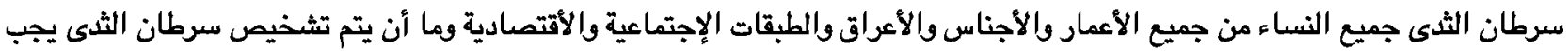

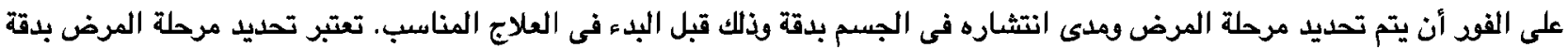

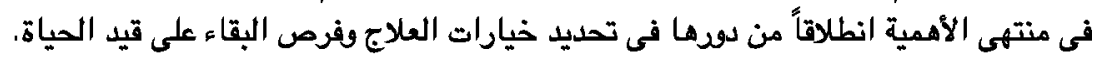

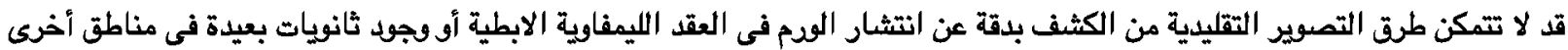

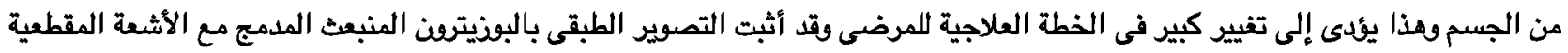

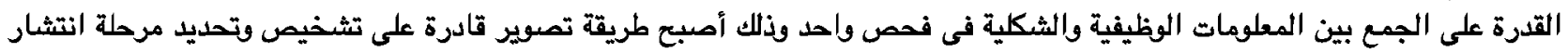

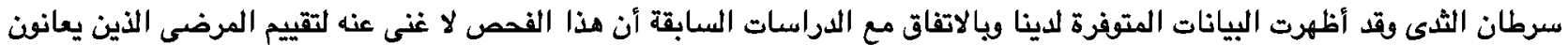

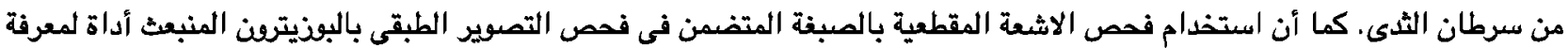

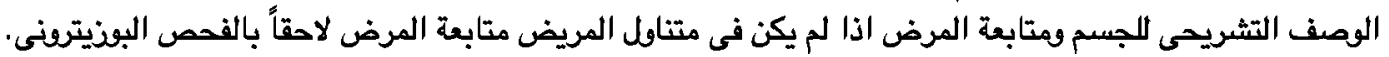

